\title{
Modulatory Role of Drebrin on the Cytoskeleton within Dendritic Spines in the Rat Cerebral Cortex
}

\author{
Kensuke Hayashi, ${ }^{1}$ Ryoki Ishikawa, ${ }^{2}$ Li-Hong Ye, ${ }^{2}$ Xiao-Ling He, ${ }^{1}$ Kuniaki Takata, ${ }^{3}$ Kazuhiro Kohama, ${ }^{2}$ and \\ Tomoaki Shirao ${ }^{1}$ \\ Departments of ${ }^{1}$ Neurobiology and Behavior and ${ }^{2}$ Pharmacology, Gunma University School of Medicine, and ${ }^{3}$ Laboratory \\ of Molecular and Cellular Morphology, Institute for Molecular and Cellular Regulation, Gunma University, Maebashi, \\ Gunma 371, Japan
}

\begin{abstract}
Morphological changes in the dendritic spines have been postulated to participate in the expression of synaptic plasticity. The cytoskeleton is likely to play a key role in regulating spine structure. Here we examine the molecular mechanisms responsible for the changes in spine morphology, focusing on drebrin, an actin-binding protein that is known to change the properties of actin filaments. We found that adult-type drebrin is localized in the dendritic spines of rat forebrain neurons, where it binds to the cytoskeleton. To identify the cytoskeletal proteins that associated with drebrin, we isolated drebrin-containing cytoskel-
\end{abstract}

etons using immunoprecipitation with a drebrin antibody. Drebrin, actin, myosin, and gelsolin were co-precipitated. We next examined the effect of drebrin on actomyosin interaction. In vitro, drebrin reduced the sliding velocity of actin filaments on immobilized myosin and inhibited the actin-activated ATPase activity of myosin. These results suggest that drebrin may modulate the actomyosin interaction within spines and may play a role in the structure-based plasticity of synapses.

Key words: spine; synapse; cytoskeleton; plasticity; actomyosin; drebrin
A dendritic spine is a dynamic structure that is extremely responsive to changes in ambient conditions (for review, see Koch and Zador, 1993). The shape of spines changes during neuronal development (Harris and Stevens, 1989; Harris et al., 1992; Papa et al., 1995), as well as in response to behavioral or environmental cues (Purpura, 1974; Coss and Globus, 1978; Bradley and Horn, 1979; Brandon and Coss, 1982). Several studies have suggested a role for the morphology of spines in the induction and retention of synaptic plasticity. Spines or postsynaptic densities are reported to undergo morphological changes concurrently with long-term potentiation (LTP) of synaptic transmission (Van Harreveld and Fifkova, 1975; Fifkova and Van Harreveld, 1977; Lee et al., 1980; Chang and Greenough, 1984; Desmond and Levy, 1986; Hosokawa et al., 1995). Furthermore, computational modeling of calcium dynamics has revealed that the changes in spine geometry can significantly influence calcium signaling in the spine (Gold and Bear, 1994).

The cytoskeleton is likely to play an important role in regulating spine structure. Actin filaments are the major cytoskeletal component of dendritic spines, whereas microtubules and neurofilaments are virtually absent (Westrum et al., 1980; Landis and Reese, 1983). The actin filaments in the neck of a spine are longitudinally oriented, whereas those in the head are arranged in a lattice (Fifkova and Delay, 1982). This organization suggests

Received Feb. 22, 1996; revised Aug. 29, 1996; accepted Aug. 30, 1996.

This research was supported in part by a grant from Nissan Science Foundation and Grants-in-Aid (07780692, 08780737, and 07279107 for Scientific Research on Priority Areas on "Functional Development of Neural Circuits") from the Ministry of Education, Science, and Culture of Japan. We thank Dr. Kunihiko Obata (National Institute for Physiological Science) and Dr. George Augustine (Duke University Medical Center) for their valuable suggestions and critical reading of this manuscript.

Correspondence should be addressed to Kensuke Hayashi, Department of Neurobiology and Behavior, Gunma University School of Medicine, 3-39-22 Showamachi, Maebashi, Gunma 371, Japan.

Copyright (C) 1996 Society for Neuroscience $0270-6474 / 96 / 167161-10 \$ 05.00 / 0$ that actin filaments might provide the basic structural scaffolding of the spine. Because myosin is also present at high levels in spines (Drenckhahn and Kaiser, 1983; Morales and Fifkova, 1989), actomyosin-based motility may be responsible for changes in spine morphology.

Drebrin is an actin-binding protein expressed mainly in neurons (Shirao and Obata, 1985; Ishikawa et al., 1994). It could regulate the spine cytoskeleton: a study in the chicken brain revealed that postsynaptic sites of dendrites are rich in drebrin (Shirao et al., 1987). Drebrin was first described as a developmentally regulated brain protein whose expression is maximal during embryogenesis and decreases thereafter (for review, see Shirao, 1995). In the embryo, drebrin is accumulated in the somata of migrating neurons and in neurite processes of postmigratory neurons. The overexpression of drebrin in cultured fibroblasts causes the dissociation of tropomyosin from actin filaments and dramatic changes in stress fibers (Ishikawa et al., 1994; Shirao et al., 1994). In some cases, drebrin overexpression also yields dendrite-like cell processes (Shirao et al., 1992). Thus, drebrin seems to play an important role in neuronal morphogenesis.

In the adult brain, an embryonic type of drebrin is replaced by an adult type by alternative RNA splicing mechanisms (Kojima et al., 1993). In this study, we clarified the localization of an adult isoform of drebrin in detail in the rat brain and investigated its interaction with the cytoskeleton of spines. We find that drebrin is particularly enriched in dendritic spines and that this protein inhibits actomyosin interaction in vitro. These results are consistent with an involvement of this protein in maintaining or modifying spine structure.

\section{MATERIALS AND METHODS}

Antibodies. Antibodies were obtained from various sources. Monoclonal antibodies against drebrin, M2F6, and M2H1 (Shirao and Obata, 1986) and against synaptophysin/SVP38, namely 171B5 (Obata et al., 1986), were used as culture supernatants of hybridoma cells that secreted the 
respective antibodies. Polyclonal antibodies against drebrin were raised against purified drebrin from the rat in a rabbit (Ishikawa et al., 1994). Monoclonal antibodies against MAP2 (Sigma, St. Louis, MO), $\alpha$-fodrin (Biohit, Helsinki, Finland), gelsolin (Sigma), and tropomyosin (Sigma), and polyclonal antibodies against bovine uterine myosin (Biomedical Technologies, Stoughton, MA) were purchased as indicated. Monoclonal antibodies against caldesmon and fascin were gifts from Dr. F. Matsumura (Rutgers University), and a polyclonal antibody against $\alpha$-actinin were a gift from Dr. K. Maruyama (National Institute for Physiological Sciences).

Immunohistochemistry. Ten-week-old Wistar rats were perfused with $4 \%$ paraformaldehyde in $0.1 \mathrm{M}$ phosphate buffer, $\mathrm{pH}$ 7.2. Each brain was excised and immersed overnight in the same fixative. Cryosections, $10 \mu \mathrm{m}$ thick, were treated with $0.1 \%$ Triton X-100 in PBS for $30 \mathrm{~min}$ and incubated with $3 \%$ bovine serum albumin (BSA) in PBS for $>1 \mathrm{hr}$. They were then incubated with the first antibody for $1 \mathrm{hr}$, washed with PBS for $30 \mathrm{~min}$, incubated with the second antibody (peroxidase- or fluoresceinconjugated IgG against mouse IgG; Cappel, West Chester, PA) for $1 \mathrm{hr}$ and washed for $30 \mathrm{~min}$. The immunoreaction was visualized with $3,3^{\prime}$ diaminobenzidine (DAB) or was observed with an epifluorescence microscope.

For double-immunostaining of drebrin and synaptophysin, permeabilized and BSA-treated sections were incubated with a mixture of rabbit antiserum against drebrin and a monoclonal antibody against synaptophysin. The second antibody was a mixture of rhodamine-conjugated antibodies against rabbit IgG (Cappel) and FITC-conjugated antibodies against mouse IgG (Tago, Burlingame, CA). Specimens were observed with a confocal laser microscope (MRC600; Bio-Rad, Richmond, CA) (objective lens, $100 \times$; pinhole, size 10; zoom, $5 \times$; Kahlman 8; contrast stretch factors, 1-3).

Electrophoresis and immunoblotting. SDS-PAGE was performed as described by Laemmli (1970). Gels were stained with 2D-Silver Stain II (Daiichi Pure Chemicals, Tokyo, Japan). For immunoblotting, the separated proteins were blotted on an Immobilon Transfer Membrane (Millipore, MA). The membranes were incubated in skim milk for $>4 \mathrm{hr}$ and subsequently with the first antibody for $1 \mathrm{hr}$. After they were washed in PBS for $30 \mathrm{~min}$, they were incubated with the second antibody (peroxidase-conjugated goat IgG against rabbit or mouse IgG; Cappel) for $1 \mathrm{hr}$, washed again, and incubated with DAB solution as indicated above.

Cortical neuronal culture. Primary cortical cultures were prepared as follows. Cerebral cortices were dissected from 20 -d-old fetal rats and treated with $9 \mathrm{U} / \mathrm{ml}$ papain (Worthington Biochemical, Freehold, NJ) for $20 \mathrm{~min}$, followed by trituration with a pipette. Dissociated cells were plated on a polyethylenimine-coated culture dish in DMEM containing $4.5 \mathrm{~g} / 1$ glucose, $5 \% \mathrm{FBS}$, and $5 \%$ horse serum. After $5 \mathrm{~d}$, the medium was changed to one containing $5 \mu \mathrm{M}$ cytosine arabinoside. Cultures were fed twice a week by changing half of the medium with fresh medium. Four-week-old cultures were fixed with $4 \%$ paraformaldehyde in $0.1 \mathrm{M}$ phosphate buffer, $\mathrm{pH} 7.2$, and immunostained with a monoclonal antibody against drebrin, as described above.

Postembedding immunoelectron microscopy. The procedure of Morales and Fifkova (1989) was used for postembedding immunoelectron microscopy. In brief, rats were perfused with $1 \%$ glutaraldehyde and $4 \%$ paraformaldehyde in $0.065 \mathrm{M} \mathrm{NaH}_{2} \mathrm{PO}_{4}, 1 \mathrm{mM} \mathrm{EGTA,} 5 \mathrm{mM} \mathrm{MgCl}$, pH 6.8 , and then with fixative, as described above, but with $0.5 \%$ instead of $1 \%$ glutaraldehyde. Blocks were prepared and were immersed in the final fixative for $15 \mathrm{hr}$ at $4^{\circ} \mathrm{C}$. After they were rinsed in buffer and subsequently in distilled water, blocks were treated with $1 \%$ aqueous uranyl acetate for $1.5 \mathrm{hr}$ at $4^{\circ} \mathrm{C}$. They were dehydrated in the presence of $1 \%$ uranyl acetate in an ethanol series and infiltrated with LR White (London Resin Co., Ltd., Basingstoke, UK). Polymerization was achieved by adding the accelerator and incubating the resin at $4^{\circ} \mathrm{C}$ overnight.

Ultrathin silver sections were incubated in $20 \mathrm{mM}$ Tris-buffered saline (TBS), pH 8.2, with $0.1 \%$ BSA and $5 \%$ normal horse serum for $30 \mathrm{~min}$. They were then incubated with the first antibody for $2 \mathrm{hr}$. After they were washed in $0.1 \%$ BSA in TBS, they were incubated with the second antibody conjugated with $5 \mathrm{~nm}$ colloidal gold particles (BioCell, Cardiff, $\mathrm{UK}$ ) for $2 \mathrm{hr}$. After they were washed in $0.1 \%$ BSA in TBS and in distilled water, they were fixed with $2 \%$ glutaraldehyde for 15 min and stained with $5 \%$ uranyl acetate at room temperature for $45 \mathrm{~min}$. The sections were observed with an electron microscope (2000CX; Japan Electron Optics Laboratory Co., Ltd., Tokyo, Japan).

Nineteen drebrin-stained and 11 synaptophysin-stained synapses that were labeled with $>10$ gold particles were photographed, and the particles on pre- and postsynaptic sites were counted.

Preparation of synaptosomes and observations by electron microscopy. Synaptosomes were prepared by the method of Cohen et al. (1977). Rat cerebral cortex was homogenized in 9 vol of $5 \mathrm{~mm}$ phosphate buffer and $1 \mathrm{~mm}$ EDTA with protease inhibitors ( $1 \mu \mathrm{M}$ leupeptin, $250 \mu \mathrm{M}$ PMSF, 2 $\mu \mathrm{M}$ pepstatin) and centrifuged at $1,500 \times g$ for $10 \mathrm{~min}$. The supernatant was centrifuged at $17,500 \times g$ for $20 \mathrm{~min}$. The resulting pellet was homogenized with $0.32 \mathrm{~m}$ sucrose and layered onto a discontinuous sucrose gradient. The gradient contained 1.2, 1.0, and $0.85 \mathrm{~m}$ sucrose and was centrifuged at $100,000 \times g$ for $2 \mathrm{hr}$. The synaptosomal fraction at the $1.0-1.2 \mathrm{M}$ interface was collected.

For electron microscopy, we used the method described by Kennedy et al. (1990). Synaptosomes were fixed with 9 vol of $4 \%$ paraformaldehyde, $0.1 \%$ glutaraldehyde, $0.32 \mathrm{~m}$ sucrose, and $5 \mathrm{~mm}$ phosphate buffer on ice for $30 \mathrm{~min}$. The fixed synaptosomes were recovered by centrifugation and resuspended by homogenization in a small volume of $0.12 \mathrm{M}$ phosphate buffer. The suspension was mixed with an equal volume of $3 \%$ lowmelting-point agarose in $5 \mathrm{~mm}$ phosphate buffer at $56^{\circ} \mathrm{C}$, and the mixture was quickly placed in warm frames constructed according to the methods of DeCamilli et al. (1983). The resulting thin slabs of agarose-embedded synaptosomes were cut into small blocks. The blocks were incubated in $0.5 \mathrm{M}$ Tris- $\mathrm{HCl}, \mathrm{pH} 7.4$, for $30 \mathrm{~min}$, washed in PBS, and incubated in 5\% horse serum, $3 \%$ BSA in PBS for $40 \mathrm{~min}$. The blocks were then incubated in an antiserum against drebrin or preimmune serum that had been diluted in 3\% BSA in PBS at $4^{\circ} \mathrm{C}$ overnight. They were washed in PBS for $3 \mathrm{hr}$, incubated with $5 \mathrm{~nm}$ colloidal gold-conjugated second antibody for $2 \mathrm{hr}$, washed again, and fixed in $1 \%$ glutaraldehyde, $0.12 \mathrm{~m}$ phosphate buffer for $30 \mathrm{~min}$ at $4^{\circ} \mathrm{C}$. They were then incubated in an ice-cold $1 \%$ solution of $\mathrm{OsO}_{4}$ for $1 \mathrm{hr}$, dehydrated in a graded ethanol series, and embedded in epoxy resin by standard methods. Ultrathin sections were stained with uranyl acetate for $8 \mathrm{~min}$ and observed with an electron microscope.

Fractionation of a brain homogenate and isolation of drebrin-containing cytoskeletons. For fractionation of a brain homogenate by centrifugation, fresh brains were homogenized in 9 vol of $5 \mathrm{~mm}$ Tris- $\mathrm{HCl}, \mathrm{pH} 7.5,150 \mathrm{~mm}$ $\mathrm{NaCl}, 0.5 \mathrm{~mm}$ DTT, $1 \% \mathrm{NP} 40$, and protease inhibitors (1 $\mu \mathrm{M}$ leupeptin, $250 \mu \mathrm{M}$ PMSF, $2 \mu \mathrm{M}$ pepstatin) by 10 strokes with a Teflon-glass homogenizer. The homogenate was centrifuged at $16,000 \times g$ for $20 \mathrm{~min}$ and subsequently at $100,000 \times g$ for $2 \mathrm{hr}$. The supernatant and the pellet after each centrifugation were subjected to immunoblotting.

Immunoaffinity beads were prepared as follows. Ascites fluid was collected from nude mice that had been injected with hybridoma cells that produced a monoclonal antibody. IgG was cross-linked to protein A-Sepharose 4FF (Pharmacia, Broma, Sweden) with dimethylpymelimidate, as described by Harlow and Lane (1988). The supernatant after centrifugation at $16,000 \times g$ of a homogenate of cerebral cortex, prepared as described above, was incubated with the immunoaffinity beads at $4^{\circ} \mathrm{C}$ for $2-3 \mathrm{hr}$. The beads were recovered by centrifugation at $100 \times g$ for 30 sec and washed with the homogenization buffer three times for a total 30 min. The proteins that had bound to the beads were analyzed by SDSPAGE after the beads were boiled in Laemmli's sample buffer.

In vitro motility assay. Sliding velosity of actin filaments on immobilized myosin was measured accordingly to Okagaki et al. (1991). Actin filaments were prepared by polymerization of actin that was purified from acetone powder of chicken skeletal muscle. Myosin was prepared from chicken gizzard smooth muscle and phosphorylated with myosin light chain kinase, and then it was used as myosin. Actin filaments were labeled with rhodamine-phalloidin and were allowed to move on myosin that had been fixed to a nitrocellulose-coated glass surface. Actin filaments ( $3 \mathrm{nM}$ ) with the fluorescent label were incubated in a solution of $30 \mathrm{mM} \mathrm{KCl}, 2$ mм ATP, $1 \mathrm{~mm} \mathrm{MgCl}_{2}, 25 \mathrm{~mm}$ DTT, $4.5 \mathrm{mg} / \mathrm{ml}$ glucose, $0.22 \mathrm{mg} / \mathrm{ml}$ glucose oxidase, $0.036 \mathrm{mg} / \mathrm{ml}$ catalase, and $20 \mathrm{~mm}$ imidazole- $\mathrm{HCl}, \mathrm{pH} 7.0$, in the presence or absence of $6 \mathrm{nM}$ drebrin. The movements of the actin filaments were monitored under a fluorescence microscope equipped with a video camera (C2400; Hamamatsu Photonics, Hamamatsu, Japan). Actin filaments that did not move were omitted. Twenty filaments of each experiment were monitored, and the measurements were displayed in a histogram.

Assay of ATPase activity. ATPase activity was determined by a malachite-green method modified by Kodama et al. (1986). Myosin (0.05 $\mu \mathrm{M}), 2 \mu \mathrm{M}$ actin filaments, and $0.5 \mathrm{mM}$ ATP were incubated in $20 \mathrm{mM}$ Tris- $\mathrm{HCl}, \mathrm{pH} 7.5,5 \mathrm{mM} \mathrm{MgCl}_{2}$, and $0.1 \mathrm{~mm}$ EGTA at $25^{\circ} \mathrm{C}$ for $10 \mathrm{~min}$. The hydrolysis was stopped by the addition of an equal volume of $0.6 \mathrm{M}$ perchloric acid. The mixture was centrifuged, and the amount of inor- 

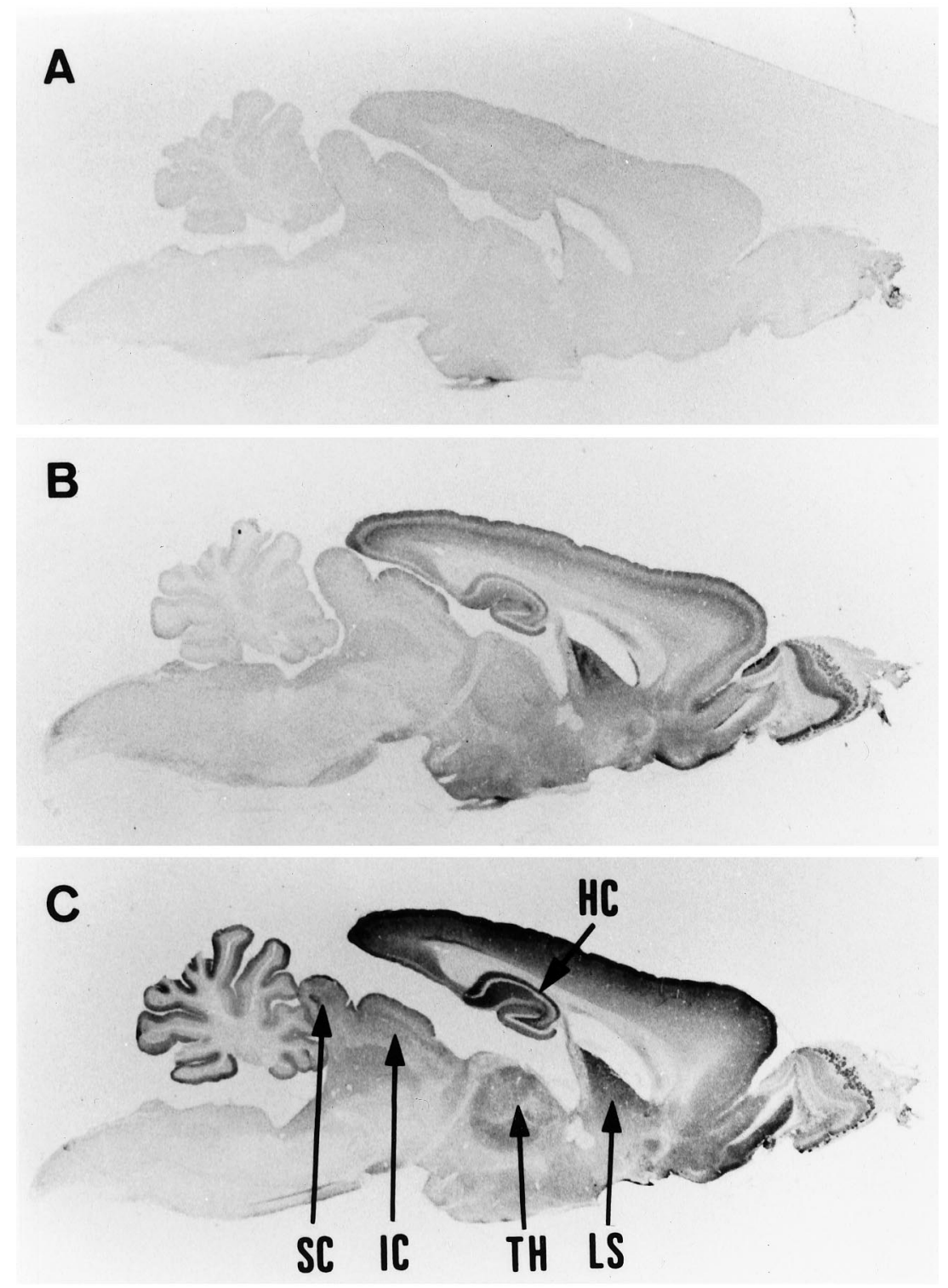

Figure 1. Adjacent sagittal sections of the brain of a 10 -week-old rat were stained with monoclonal antibodies against drebrin $(B)$ and synaptophysin $(C) . A$ shows the negative control staining without the first antibody. The gray matter of the olfactory bulb, cerebral cortex, hippocampus, and lateral septum were intensely stained with an antibody against drebrin. $H C$, Hippocampus; $L S$, lateral septum; $I C$, inferior colliculus; $S C$, superior colliculus; $T H$, thalamus. ganic phosphate in each supernatant was determined by measuring the absorbance at $650 \mathrm{~nm}$ after incubation with the malachite-green reagent. Assays were carried out in the presence of $0,0.25,0.5$, and $1.0 \mu \mathrm{M}$ purified drebrin.

\section{RESULTS}

\section{The rat forebrain is rich in adult-type drebrin}

We first studied the distribution of drebrin in CNS of adult rats by an immunohistochemical method (Fig. 1). A monoclonal antibody against drebrin (M2F6) stained gray matter of the brain (Fig. 1B), which was also stained with an antibody against synaptophysin (Fig. $1 C$ ). The intensity of immunostaining with an antibody against drebrin, however, was not exactly correlated with the intensity with an antibody against synaptophysin. For example, the cerebral cortex, hippocampus, lateral septum, and striatum (not shown) were intensely stained with both antibodies, whereas the staining in the inferior and superior colliculus and cerebellar cortex was weak, with an antibody against drebrin compared with the staining with an antibody against synaptophysin (compare Fig. $1 B$ with $1 C$ ).

Immunohistochemical data provide no information about the relative distribution of drebrin isoforms, because the antibody reacts with both the embryonic (drebrin E) and adult (drebrin A) isoforms of drebrin. To distinguish between these forms, we performed immunoblot analysis (Fig. $2 A$ ). It showed that drebrin $\mathrm{E}$ (bottom band) was uniformly distributed at a low level throughout the brain, with the exception of its abundant expression in olfactory bulb (lane 1). By contrast, drebrin A (top band) was expressed differentially, being abundant in the cerebral cortex and hippocampus, but present only at low levels in cerebellar cortex, pons, medulla, and spinal cord.

We next studied developmental changes in the expression of drebrin (Fig. $2 B$ ). The expression of drebrin $E$ decreased at $\sim 4$ weeks after birth, with a similar time course in both the cerebral and cerebellar cortices. Drebrin A was expressed at 1 week after birth in the cerebral cortex, and its level increased thereafter. In the cerebellar cortex, although a small amount of drebrin A was detected $>4$ weeks after birth, it remained at a level lower than that of drebrin $\mathrm{E}$ at least until 10 weeks after birth. The time course of developmental expression of drebrin A in the cerebral cortex was very similar to that of synaptophysin, indicating that 

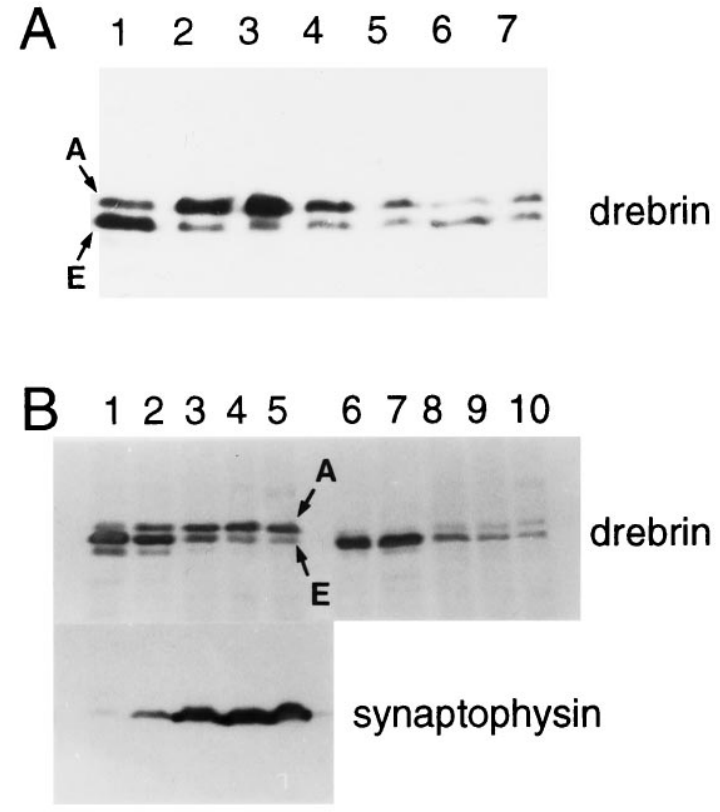

Figure 2. Immunoblotting analysis of the expression of drebrin isoforms in the rat brain. $A$, Drebrin A ( $A$, top band) was expressed heterogenously in the adult rat brain, whereas embryonic type ( $E$, bottom band) was distributed uniformly at a low level, except in olfactory bulb. Total protein from the following tissues of a 10 -week-old rat, normalized in each case with respect to wet weight, were subjected to SDS-PAGE and immunostaining with a monoclonal antibody against drebrin (M2F6). Lane 1, Olfactory bulb; lane 2, cerebral cortex; lane 3, hippocampus; and lane 4, rostral half of the remainder of the brain after removal of the olfactory bulb, cerebral cortex, hippocampus, and cerebellar cortex. This region included the striatum, thalamus, and midbrain. Lane 5, Caudal half of the remainder including pons and medulla; lane 6 , cerebellar cortex; and lane 7 , spinal cord. $B$, The time course of developmental expression of drebrin A (top band) in the cerebral cortex was very similar to that of synaptophysin. Total protein from the cerebral cortex (lanes 1-5) or the cerebellar cortex (lanes 6-10) of a 1-week-old (lanes 1, 6), 2-week-old (lanes 2, 7), 4-week-old (lanes 3, 8), 6-week-old (lanes 4, 9), and 10-week-old (lanes 5, 10) rat, respectively, was subjected to SDS-PAGE and immunostaining with an antibody against drebrin (M2F6) or an antibody against synaptophysin.

drebrin A is expressed coincidentally with the formation of synapses.

\section{Drebrin is localized at postsynaptic sites}

The products of the immunohistochemical staining of the cerebral cortex with an antibody against drebrin were seen as scattered dots (Fig. 3B), similar to the appearance of the staining obtained with the synaptophysin antibody, which was used to visualize synapses (Fig. 3C). Dendrites and somata of neurons were not stained with an antibody against drebrin, whereas both were stained with an antibody against MAP2 (Fig. 3D). When primarycultured cortical neurons were stained with an antibody against drebrin, intense staining was observed as scattered dots attached to dendrites and cell body (Fig. $3 E$ ). These suggest that drebrin was localized at synapses.

To clarify the location of drebrin, a postembedding immunoelectron microscopic study was performed. Drebrin was detected mainly at the postsynaptic sites of synapses in the cerebral cortex (Fig. 4A-C) and in the hippocampus (data not shown); $91.3 \%$ of the immunogold particles on clearly identified synapses (19 synapses were analyzed) were at the postsynaptic sites. Drebrin immunolabeling was evenly distributed within spines and not condensed at the postsynaptic density. By contrast, as expected, synaptophysin was observed at presynaptic sites $(95.4 \%$ of the particles on 11 synapses analyzed; Fig. 4D).

The high concentration and strict localization of drebrin in spines enabled us to distinguish spines in brain sections by confocal laser scanning microscopy (Fig. 5). Double-labeling of drebrin and synaptophysin of a cerebral cortex section showed a scattered dot pattern of staining with both antibodies (Fig. 5A). High-power magnification showed a clear separation between synaptophysin-labeled presynaptic terminals and drebrin-labeled postsynaptic spines (Fig. 5B). As far as we know, drebrin is one of the most strictly localized proteins in the postsynaptic spines.

\section{Drebrin binds to the cytoskeleton of spines}

To examine the association of drebrin with the cytoskeleton in vivo, we monitored the detergent extractability of drebrin in brain homogenates. Almost all of the drebrin was pelleted during centrifugations in the presence of 1\% NP40 (Fig. 6). Taken together with the fact that partially purified drebrin from the adult rat brain is soluble (Shirao et al., 1994), the failure to extract drebrin in the final supernatants suggests that drebrin binds to cytoskeleton in vivo. This mimics the behavior of other actin-binding proteins; some, such as fodrin, myosin, $\alpha$-actinin, and gelsolin, were also unextractable, although others, such as MAP2, caldesmon, fascin, and tropomyosin, were not (Fig. 6).

We next observed the localization of drebrin in synaptosomes by immunoelectron microscopy with an antibody against drebrin and colloidal gold-labeled second antibody. The immunogold particles were seen on filamentous materials that were associated with postsynaptic densities (Fig. $7 A-C$ ); $19.7 \pm 19.1$ (mean $\pm \mathrm{SD}$, $n=24$ ) particles were found on postsynaptic sites. Few particles $(2.3 \pm 2.1, n=16)$ were seen with a preimmune serum (Fig. $7 D)$. These results show directly that drebrin binds to the spine cytoskeleton, because unbound proteins in spines should be washed out during the preparation of the synaptosomes (Kennedy et al., 1990).

\section{Drebrin forms a complex with actin, myosins, and gelsolin}

To identify the cytoskeletal proteins that are associated with drebrin, we used immunoprecipitation methods. When drebrin was immunoprecipitated with a monoclonal antibody against drebrin from the rat cerebral cortex, proteins of 220, 190, and $90 \mathrm{kDa}$ and actin were co-precipitated (Fig. $8 A$ ). All of these proteins were immunoprecipitated with both M2F6 and M2H1 antibodies, which recognized different epitopes on the drebrin molecule (Shirao and Obata, 1986), but they were not immunoprecipitated by a synaptophysin antibody. The protein composition was unchanged when the cortex was homogenized with $2 \mathrm{mM} \mathrm{Ca}^{2+}$ ions or with 10 mm EGTA, with the exception that the amount of 90 $\mathrm{kDa}$ protein was reduced in the presence of EGTA (data not shown).

The 220 and $90 \mathrm{kDa}$ proteins were identified as myosin II and gelsolin, respectively, by immunoblotting. Figure $8 B$ shows the co-precipitation of myosin II, drebrin, gelsolin, and actin with an antibody against drebrin but not with an antibody against synaptophysin. The $190 \mathrm{kDa}$ and $130 \mathrm{kDa}$ proteins seemed to be myosins, because they and the $220 \mathrm{kDa}$ protein shifted into the supernatant in the presence of $0.5 \mathrm{~mm}$ ATP (Fig. 8C) (Espindola et al., 1992; Bähler et al., 1994). Based on their molecular weight, the 190 and $130 \mathrm{kDa}$ proteins seemed to be myosin V (Espreafico et al., 1992) and myosin I (Wagner et al., 1992), respectively. 

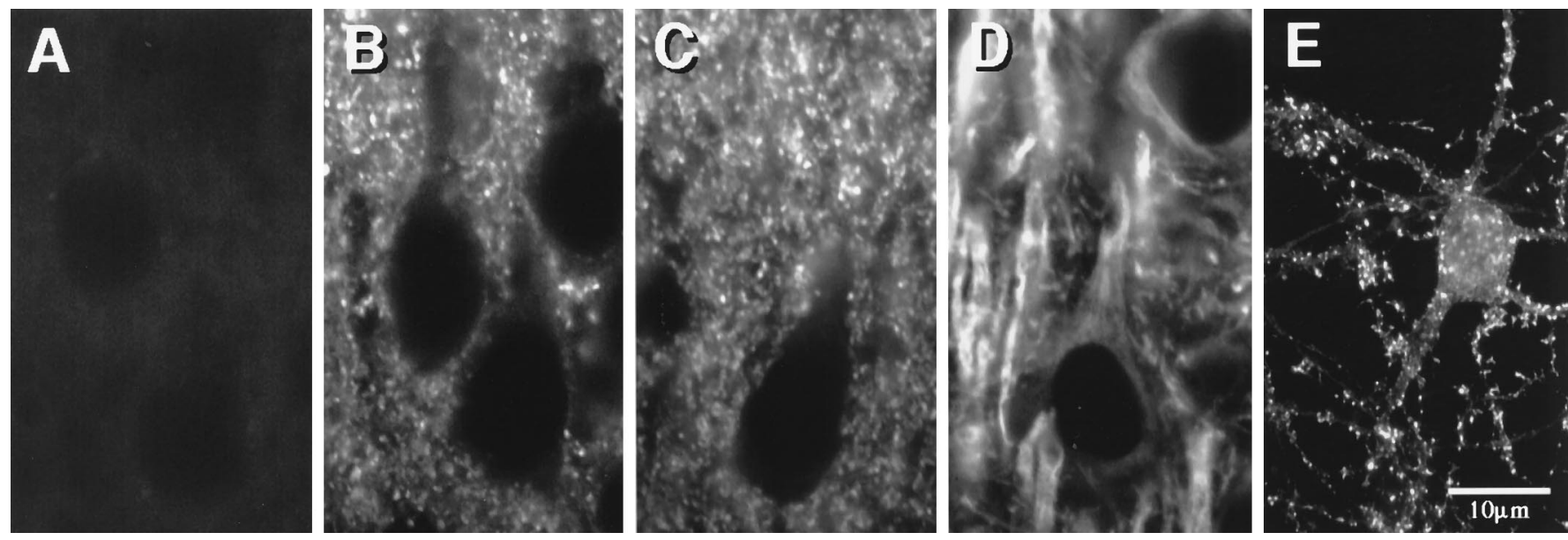

Figure 3. Sections of the cerebral cortex were stained with antibodies against drebrin $(B)$, synaptophysin $(C)$, and MAP2 $(D)$. $A$ shows the negative control staining without the first antibody; $E$ shows a primary-cultured cortical neuron stained with an antibody against drebrin. Immunostaining with drebrin and that with synaptophysin were seen as scattered dots, whereas an antibody against MAP2 stained cell bodies and dendrites. Scale bar, $10 \mu \mathrm{m}$.
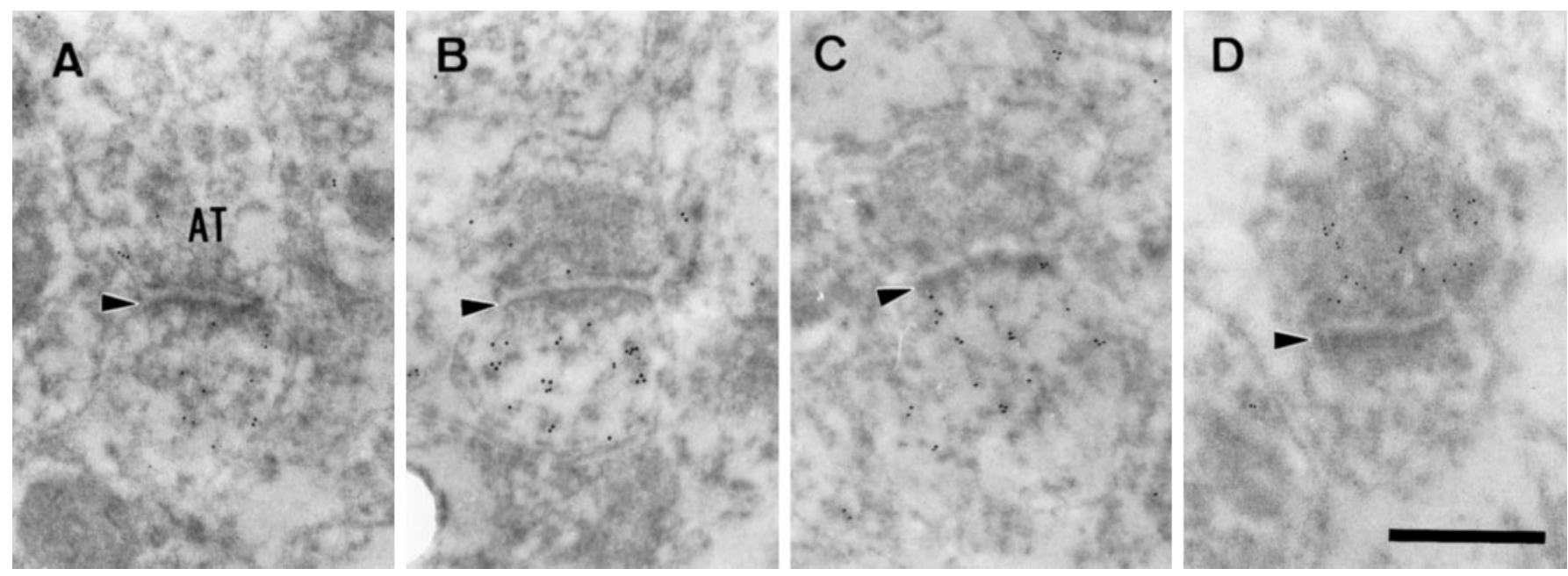

Figure 4. Postembedding immunoelectron microscopy showing the localization of drebrin in the dendritic spines. Ultrathin sections of cerebral cortex were stained with an antibody against drebrin, M2F6 $(A-C)$, and with an antibody against synaptophysin $(D)$. Arrowheads indicate postsynaptic densities. $A T$, Axon terminal. Scale bar, $1 \mu \mathrm{m}$.

MAP2, fodrin, caldesmon, $\alpha$-actinin, fascin, and tropomyosin, all of which have an actin-binding activity and are detectable in the brain, were not detected within the immunoprecipitates, even by immunoblotting (data not shown).

\section{Drebrin inhibits actomyosin interaction}

The association of drebrin with actin and myosin suggests that drebrin could regulate actomyosin interaction. This possibility was tested in two ways. First, the sliding velocity of actin filaments on myosin immobilized on a glass surface was measured with or without purified drebrin (Fig. 9A). Although actin filaments moved at an average velocity of $0.34 \mu \mathrm{m} / \mathrm{sec}$ in the absence of drebrin, the presence of drebrin (molar ratio of actin to drebrin, 1:2) reduced their sliding velocity to $0.10 \mu \mathrm{m} / \mathrm{sec}$ on average.

Second, the actin-activated ATPase activity of myosin was measured in the presence and absence of purified drebrin (Fig. 9B). Drebrin at $0.25 \mu \mathrm{M}$ (molar ratio of actin to drebrin, 8:1) inhibited the ATPase activity by $\sim 40 \%$, which may account for the ability of drebrin to slow actin filament sliding. These results demonstrate the inhibitory effect of drebrin on actomyosin interaction and suggest that drebrin is one of the actin-linked regulatory proteins.

\section{DISCUSSION}

The present study focused on the interactions of drebrin with the spine cytoskeleton and revealed three new characteristics of this protein. First, drebrin is localized in the dendritic spines in the forebrain and binds to the spine cytoskeleton; second, drebrin forms a complex with actin, myosins, and gelsolin; and third, drebrin inhibits the actomyosin interaction.

\section{Localization of drebrin in the adult rat brain}

Immunohistochemical and immunoblot analysis revealed a heterogeneous distribution of drebrin $\mathrm{A}$ in the adult rat brain. Drebrin A was more concentrated in the forebrain than in other regions, whereas drebrin $\mathrm{E}$ was distributed uniformly. This regional difference in the distribution of drebrin A may be attributable to differences in both the transcription of the gene for drebrin and the splicing of its transcripts, because the total amount of the two isoforms of drebrin, as well as the ratio of isoforms, differs among regions; however, the mechanisms of the region-dependent expression and splicing of the gene for drebrin are unknown. The forebrain is known as a region responsible for learning and mem- 

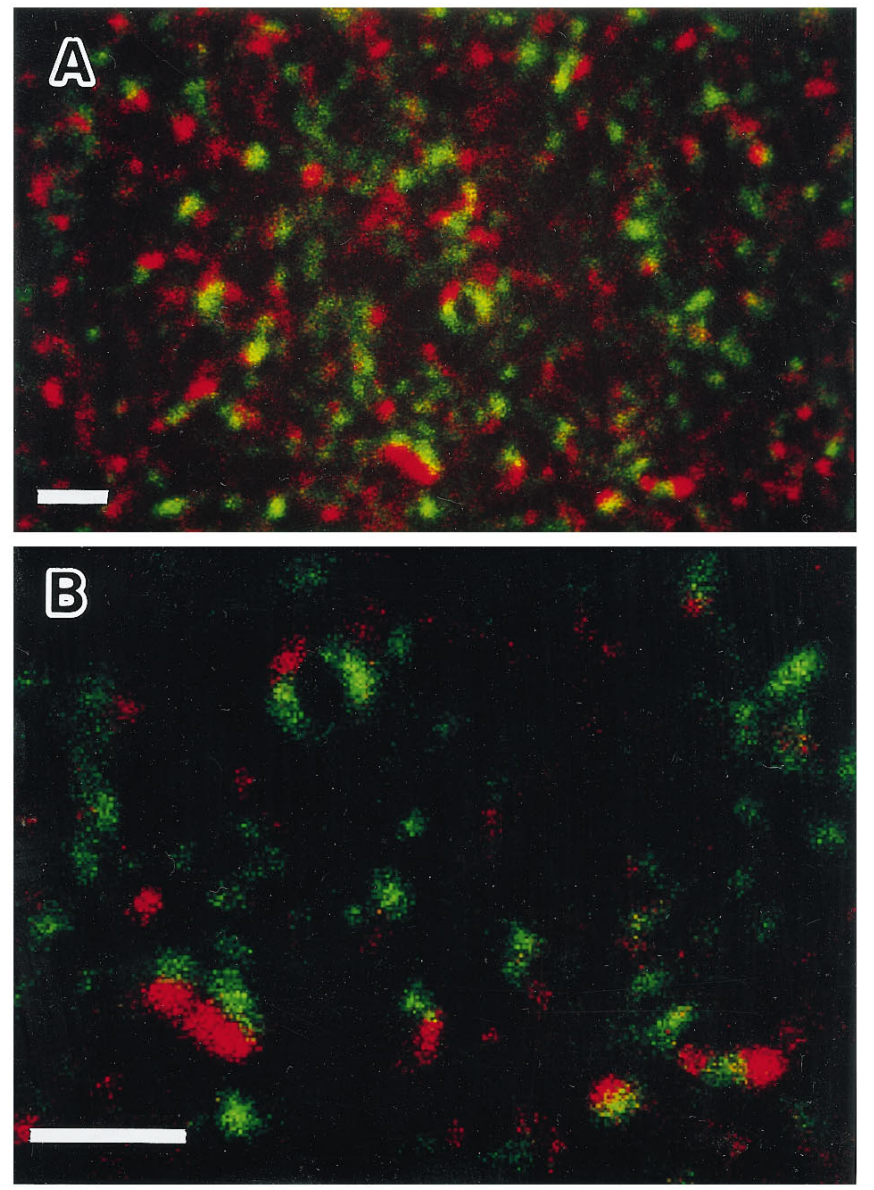

Figure 5. Confocal laser microscopic analysis of drebrin distribution in cerebral cortex. $A$, Cryosections of cerebral cortex were doubleimmunostained with an antibody against synaptophysin (green) and an antibody against drebrin (red). The immunostaining with both antibodies appeared as dots. Scale bar, $10 \mu \mathrm{m}$. $B$, High-power magnification of $A$. The staining with drebrin antibody (red) was closely adjacent to the staining with synaptophysin antibody (green). Scale bar, $1 \mu \mathrm{m}$.

ory, and the synapses in this region show typical LTP (for review, see Bliss and Collingridge, 1993). Therefore, the concentration of drebrin $\mathrm{A}$ in the forebrain suggests that the role of drebrin in adult brain is related to such characteristics of the forebrain.

Immunohistochemical staining with the antibody against drebrin was restricted to the gray matter, and at high-power magnification appeared as dots, which is consistent with our previous work (Shirao et al., 1989). The size, density, and distribution of these dots were very similar to those observed with the antibody against synaptophysin, suggesting that they represent synapses. Indeed, confocal microscopy revealed the close association of drebrin-specific dots with synaptophysin-specific dots. The sites of drebrin-specific dots were identified as dendritic spines by postembedding immunoelectron microscopy. The postsynaptic rather than presynaptic localization of drebrin was suggested by previous studies showing that drebrin but not synaptophysin disappeared in brains of patients with Alzheimer's disease (Harigaya et al., 1996) and after ischemia (Arai et al., 1991). Under both conditions, postsynaptic structures degenerate more extensively than presynaptic ones. We do not know yet whether drebrin is present at the postsynaptic sites of synapses that make direct contact with the dendritic shaft or the cell body.

We could hardly detect drebrin in dendritic shafts, cell somata,

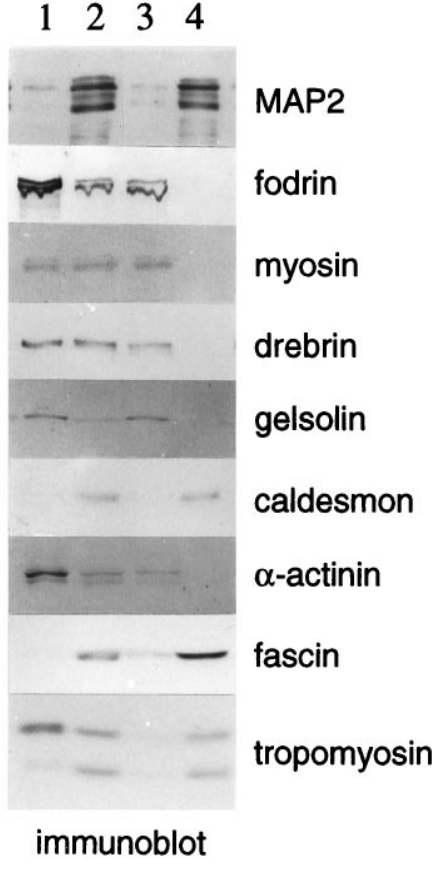

Figure 6. Detergent extractability of various actin-binding proteins in brain homogenates. Cerebral cortex of a 10 -week-old rat was homogenized in a buffer that contained 1\% NP40 and centrifuged at 16,000 $\times g$ (lane 1, pellet; lane 2, supernatant). The supernatant was subsequently centrifuged at 100,000 $\times g$ (lane 3, pellet; lane 4, supernatant). Various actin-binding proteins were examined by immunoblotting. For the detection of gelsolin, rabbit brain was used because the monoclonal antibody did not recognize rat gelsolin. Note that drebrin was not extracted in the final supernatant (lane 4). Other actin-binding proteins, such as fodrin, myosin, $\alpha$-actinin, and gelsolin, were also unextractable.

axons, or presynaptic terminals, although it is difficult to conclude that drebrin is absent there. The strict localization of drebrin in spines is striking, because most cytoskeletal proteins are distributed throughout neurons from dendrites to axons. Even MAP2, which is known to be segregated into the somatodendritic compartment, is not concentrated in the spine but rather in dendritic shafts. The major components of postsynaptic densities, CaM kinase II (Kennedy et al., 1983) and fodrin (Carlin et al., 1983), are also detected in nonsynaptic dendritic shafts, cell bodies, and axons. Furthermore, most of the receptors for neurotransmitters, such as NMDA receptors (Petralia et al., 1994a,b), AMPA glutamate receptors (Martin et al., 1993; Baude et al., 1994), and metabotropic glutamate receptors (Baude et al., 1993), are found not only at postsynaptic sites but also along dendrites and on cell bodies. Thus, we consider that drebrin is one of the most strictly localized proteins in spines. This feature might be useful as a postsynaptic molecular marker for studies of the formation or degeneration of synapses. Moreover, the strict localization of drebrin in spines in the adult brain indicates that drebrin functions mainly in spines.

\section{Drebrin-containing cytoskeleton of the spine}

Our failure to extract drebrin with detergents indicates that drebrin binds to the spine cytoskeleton in vivo. This result was confirmed by immunogold staining of drebrin on fibrous structures that protruded from postsynaptic densities of synaptosomes. Binding of drebrin to the spine cytoskeleton might influence the organization of actin filaments within the spine via the biochemical characteristics of drebrin. Previous work (Ishikawa et al., 

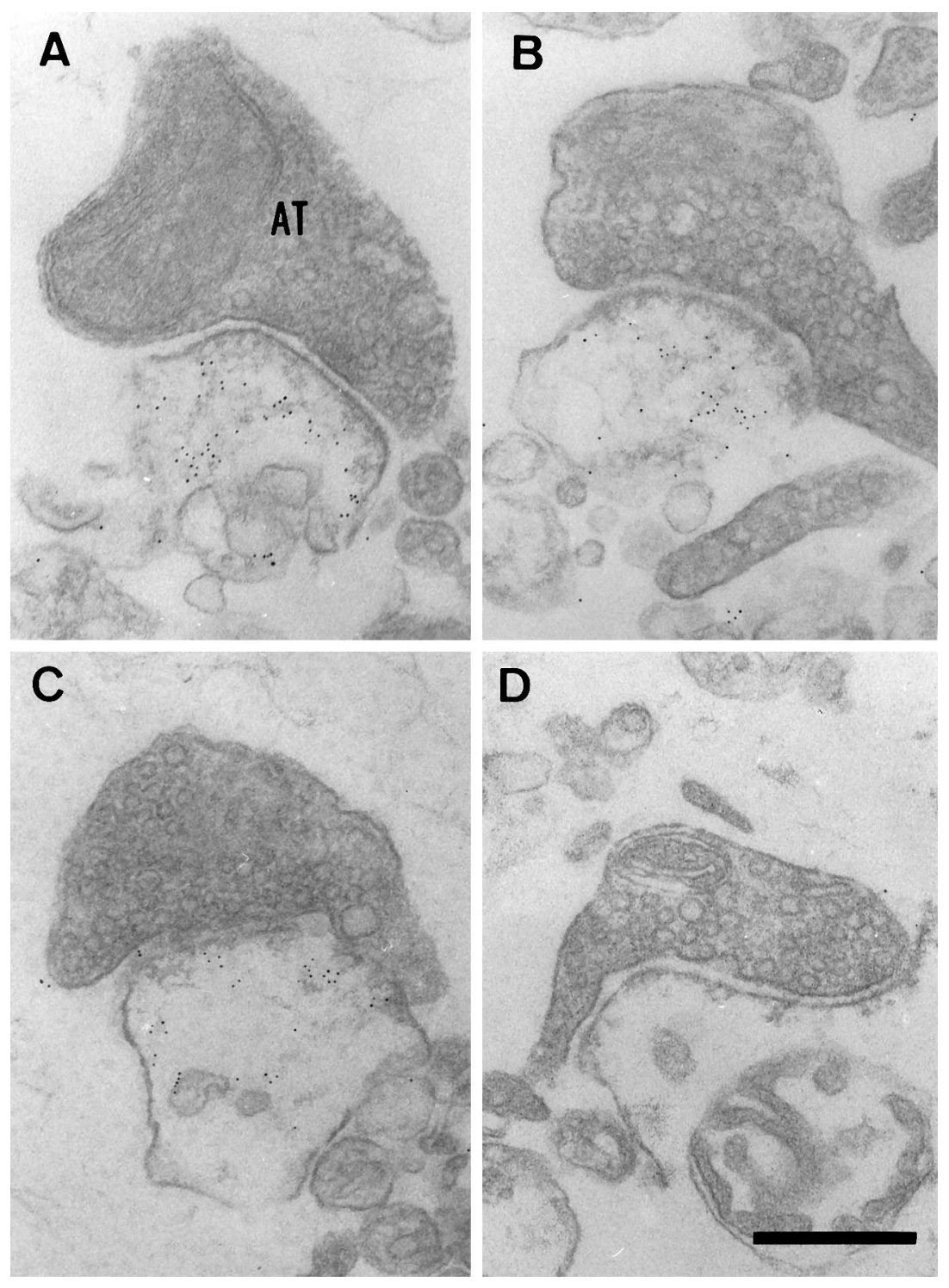

Figure 7. A synaptosome stained with an antibody against drebrin. A synaptosome fraction was prepared from the cerebral cortex of 10 -week-old rats by sucrose densitygradient centrifugation and was stained with the antibody against drebrin $(A-C)$ and with the preimmune serum $(D)$. In $A-C$, immunogold particles are visible on cytoskeletal materials associated with the postsynaptic density. $A T$, Axon terminal. Scale bar, $200 \mathrm{~nm}$.
Figure 8. The drebrin-containing cytoskeleton of the spine. $A$, Composition of the drebrin-containing cytoskeleton. The supernatant after centrifugation at $16,000 \times g$ of a homogenate in $1 \%$ NP40 of cerebral cortex was incubated with protein A-Sepharose beads that had been cross-linked with a monoclonal antibody against drebrin [M2H1 (left), M2F6 (middle)] and with a monoclonal antibody against synaptophysin (right). Proteins bound to the beads were analyzed by SDS-PAGE and silver staining. $B$, Detection of myosin II, drebrin, gelsolin, and actin in the drebrin-containing cytoskeleton by immunoblotting. The drebrin-containing cytoskeleton that was purified with immunoaffinity beads (left) and the same volume of sample from the same procedure performed with immunoaffinity beads conjugated with antibody against synaptophysin (right) were analyzed. For detection of gelsolin, rabbit brain was used because the monoclonal antibody did not recognize rat gelsolin. $C, 220,190$, and $130 \mathrm{kDa}$ proteins were eluted from drebrin-specific affinity beads by $0.5 \mathrm{~mm}$ ATP. The gel was stained with silver. Left, Proteins that bound to the beads; middle, proteins remaining after elution with ATP; right, proteins eluted with ATP.
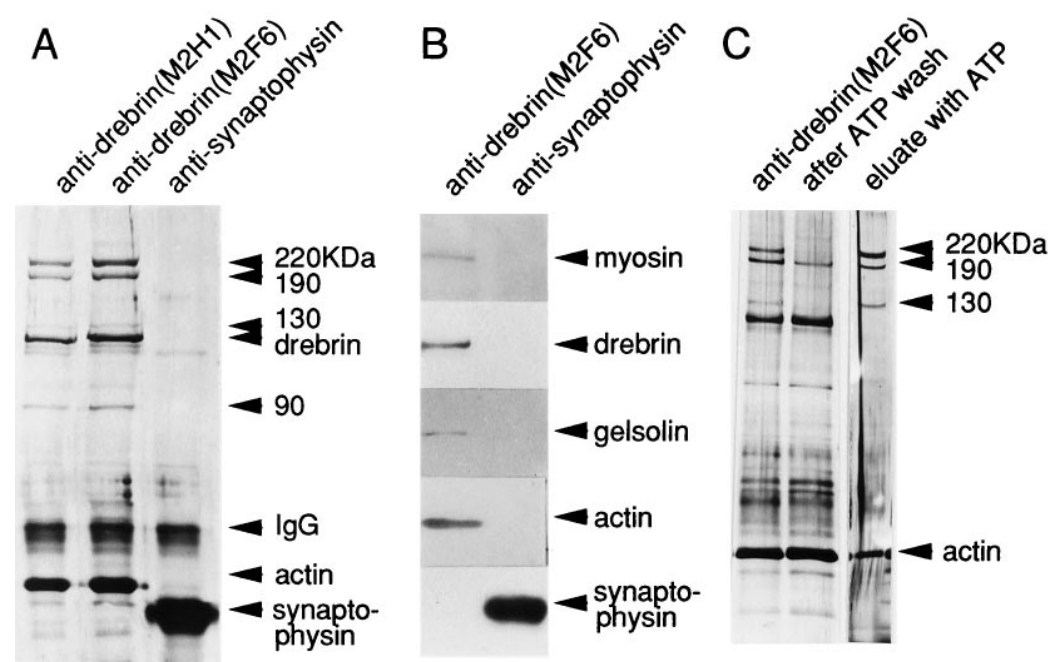

silver stain immunoblot silver stain 

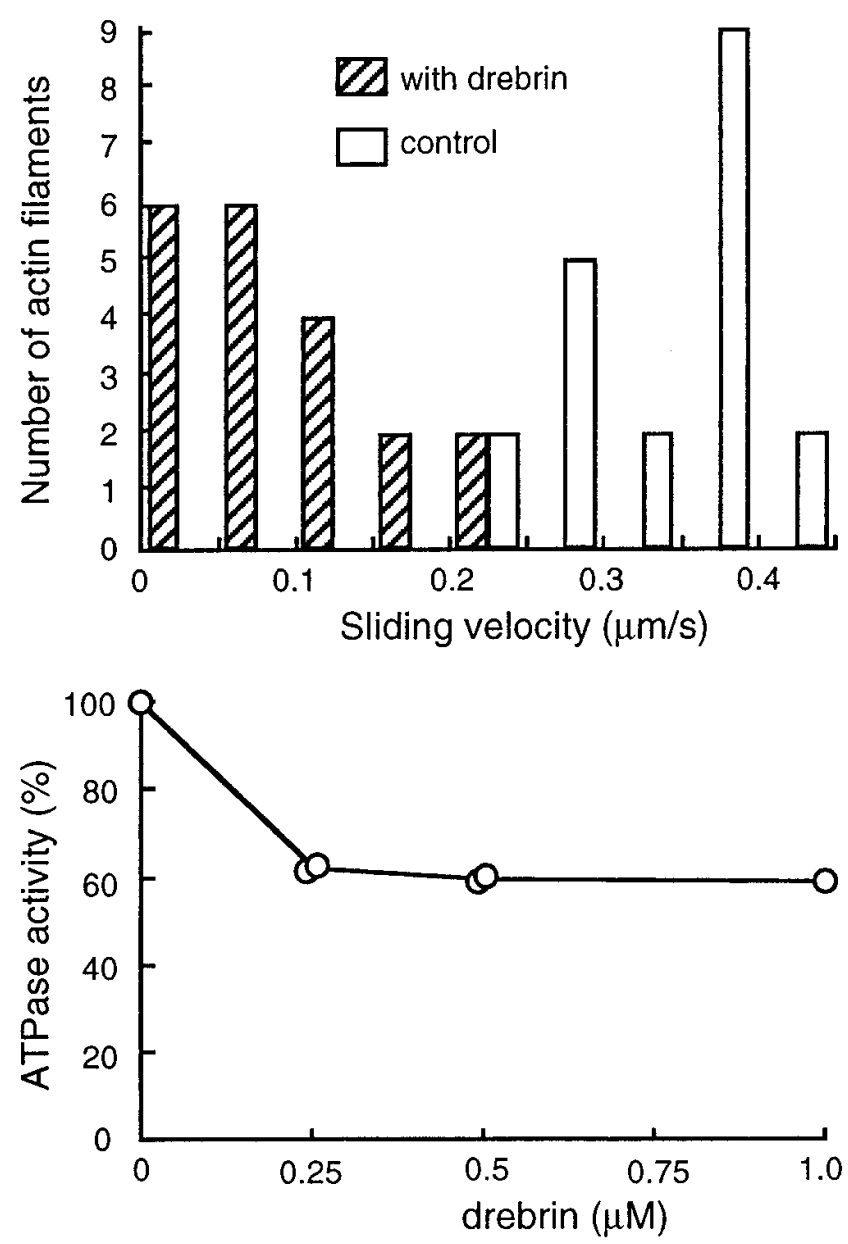

Figure 9. Inhibition of actomyosin interactions by drebrin. $A$, The motility assay revealed the inhibitory effect of drebrin on actomyosin activity. The sliding velocities of actin filaments on myosin, immobilized on a glass surface, were measured in the presence and absence of $6 \mathrm{~nm}$ purified drebrin. The velocities of 20 filaments of each experiment were measured, and the result was displayed in a histogram. $B$, Drebrin inhibited the actin-dependent ATPase activity of myosin. Myosin ATP hydrolysis was assayed by a modified malachite-green method with $2 \mu \mathrm{M}$ actin filament in the presence of purified drebrin at concentrations of $0,0.25,0.5$, and $1.0 \mu \mathrm{M}$.

1994; Sasaki et al., 1996) has demonstrated several biochemical properties of drebrin. First, drebrin binding prevents actin filaments from cross-linking. This is because drebrin binding is competitive with actin-cross-linking proteins such as $\alpha$-actinin and fascin (Ishikawa et al., 1994; Sasaki et al., 1996). Second, drebrin may destabilize actin filaments. It inhibits the actin-binding activity of tropomyosin, which protects actin filaments from severing the action of gelsolin (Ishikawa et al., 1989, 1994). Actin filaments with drebrin are accessible to the gelsolin attack. These characteristics of drebrin result in the formation of a flexible meshwork of unbundled and short actin filaments and might be responsible for the lattice-like arrangement of actin filaments in spines. The organization of actin filaments within spines should influence the shape of the spines and the morphology of postsynaptic densities and might be directly related to the control of synaptic transmission (Rosenmund and Westbrook, 1993). Thus, drebrin would help regulate postsynaptic structure and function.

To identify the components of the cytoskeleton to which dre- brin binds, we isolated the drebrin-containing cytoskeleton by immunoprecipitation. This entity contained myosins and gelsolin as well as drebrin and actin. Our failure to detect $\alpha$-actinin, tropomyosin, and fascin in the drebrin-containing cytoskeleton is consistent with the biochemical result that drebrin inhibits their actin-binding activity (Ishikawa et al., 1994; Sasaki et al., 1996). The presence of myosins in the drebrin-containing cytoskeleton was consistent with the concentration of myosin in spines (Drenckhahn and Kaiser, 1983; Morales and Fifkova, 1989). The presence of gelsolin was consistent with the biochemical result that drebrin-associated actin filaments are accessible to cleavage by gelsolin (Ishikawa et al., 1994). Although gelsolin was reported to accumulate specifically in oligodendrocytes within the brain (Tanaka and Sobue, 1994), it could be present in other types of cells in the brain, including neurons, at low levels. For example, growth cones of differentiated PC12 cells and of neurons of the rat dorsal root ganglion contain immunocytochemically detectable gelsolin (Tanaka et al., 1993). Moreover, we detected gelsolin in the drebrin-containing cytoskeleton isolated from differentiated neuroblastoma cells SY5Y (unpublished data). Thus, we consider that the gelsolin that co-precipitated with drebrin was not derived from oligodendrocytes.

\section{Modulation by drebrin of spine morphology}

The actin meshwork within the dendritic spine is thought to regulate spine morphology and to participate in the structural changes associated with the plasticity of synaptic transmission. Morales and Fifkova (1989) proposed the following hypothesis for morphological changes in spines produced by repeated stimulation. The locally increased level of $\mathrm{Ca}^{2+}$ ions within spines after repeated stimulation causes fragmentation of selective domains of the actin network through the activation of $\mathrm{Ca}^{2+}$-sensitive actinsevering protein(s) such as gelsolin. This event may reorganize the actin network in spines. The actomyosin interaction would be triggered by the elevated level of $\mathrm{Ca}^{2+}$ ions, which would activate the myosin by phosphorylation catalyzed by myosin light chain kinase (Morales and Fifkova, 1989).

Drebrin might function in the mechanism described above in two ways. First, binding of drebrin to actin filaments dissociates tropomyosin and makes them accessible to the actin-severing activity of gelsolin, enabling actin filaments to be reorganized in response to $\mathrm{Ca}^{2+}$ ions. Second, in addition to the regulatory system by myosin light chain kinase, drebrin may also regulate the actomyosin interaction. Such dual control of actomyosin interaction, namely myosin-linked and actin-linked control, has been established in some types of cells. In smooth muscle, the actinlinked control is carried out by caldesmon (Sobue and Sellers, 1991), which has an inhibitory effect on actomyosin interaction similar to that of drebrin, and has an inhibitory effect on the actin-severing activity of gelsolin (Ishikawa et al., 1989), which is different from that of drebrin. Our finding that drebrin but not caldesmon binds to actin filaments in spines suggests that drebrin takes the place of caldesmon in the actin-linked control of actomyosin interaction in spines and might play a role in the structurebased plasticity of synapses.

\section{REFERENCES}

Arai H, Sato K, Uto A, Yasumoto Y (1991) Effect of transient cerebral ischemia in mongolian gerbils on synaptic vesicle protein (SVP-38) and developmentally regulated brain protein (drebrin). Neurosci Res Commun 9:143-150. 
Bähler M, Kroschewski R, Stöffler H-E, Behrmann T (1994) Rat myr 4 defines a novel subclass of myosin I: identification, distribution, localization, and mapping of calmodulin-binding sites with differential calcium sensitivity. J Cell Biol 126:375-389.

Baude A, Nusser Z, Roberts JDB, Mulvihill E, McIlhinney RAJ, Somogyi $\mathrm{P}$ (1993) The metabotropic glutamate receptor (mGluR1 $\alpha$ ) is concentrated at perisynaptic membrane of neuronal subpopulations as detected by immunogold reaction. Neuron 11:771-787.

Baude A, Molnar E, Latawiec D, McIlhinney RAJ, Somogyi P (1994) Synaptic and nonsynaptic localization of the GluR1 subunit of the AMPA-type excitatory amino acid receptor in the rat cerebellum. J Neurosci 14:2830-2843.

Bradley P, Horn G (1979) Neuronal plasticity in the chick brain: morphological effects of visual experience on neurones in hyperstriatum accessorium. Brain Res 162:148-153.

Brandon JG, Coss RG (1982) Rapid dendritic spine stem shortening during one-trial learning: the honeybee's first orientation flight. Brain Res 252:51-61.

Bliss TVP, Collingridge GL (1993) A synaptic model of memory: longterm potentiation in the hippocampus. Nature 361:31-39.

Carlin RK, Bartelt DC, Siekevitz P (1983) Identification of fodrin as a major calmodulin-binding protein in postsynaptic density preparations. J Cell Biol 96:443-448.

Chang F-LF, Greenough WT (1984) Transient and enduring morphological correlates of synaptic activity and efficacy change in the rat hippocampal slice. Brain Res 309:34-46.

Cohen RS, Blomberg F, Berzins K, Siekevitz P (1977) The structure of postsynaptic densities isolated from dog cerebral cortex. I. Overall morphology and protein composition. J Cell Biol 74:181-203.

Coss RG, Globus A (1978) Spine stems on tectal interneurons in jewel fish are shortened by social stimulation. Science 200:787-789.

DeCamilli P, Harris Jr SM, Huttner WB, Greengard P (1983) Synapsin I (protein I), a nerve terminal-specific phosphoprotein. II. Its specific association with synaptic vesicles demonstrated by immunocytochemistry in agarose-embedded synaptosomes. J Cell Biol 96:1355-1373.

Desmond NL, Levy WB (1986) Changes in the numerical density of synaptic contacts with long-term potentiation in the hippocampal dentate gyrus. J Comp Neurol 253:466-475.

Drenckhahn D, Kaiser H-W (1983) Evidence for the concentration of F-actin and myosin in synapses and in the plasmalemmal zone of axons. Eur J Cell Biol 31:235-240.

Espindola FS, Espreafico EM, Coelho MV, Martins AR, Costa FRC, Mooseker MS, Larson RE (1992) Biochemical and immunological characterization of p190-calmodulin complex from vertebrate brain: a novel calmodulin-binding myosin. J Cell Biol 118:359-368.

Espreafico EM, Cheney RE, Matteoli M, Nascimento A-AC, DeCamilli PV, Larson RE, Mooseker MS (1992) Primary structure and cellular localization of chicken brain myosin-V (p190), an unconventional myosin, with calmodulin light chains. J Cell Biol 119:1541-1557.

Fifkova E, Van Harreveld A (1977) Long-lasting morphological changes in dendritic spines of dentate granular cells following stimulation of the entorhinal area. J Neurocytol 6:211-230.

Fifkova E, Delay RJ (1982) Cytoplasmic actin in neuronal processes as a possible mediator of synaptic plasticity. J Cell Biol 95:345-350.

Gold JI, Bear MF (1994) A model of dendritic spine $\mathrm{Ca}^{2+}$ concentration exploring possible bases for a sliding synaptic modification threshold. Proc Natl Acad Sci USA 91:3941-3945.

Harigaya Y, Shoji M, Shirao T, Hirai S (1996) Disappearance of actinbinding protein, drebrin, from hippocampal synapses in Alzheimer's disease. J Neurosci Res 43:87-92.

Harlow E, Lane D (1988) Antibodies, a laboratory manual. Cold Spring Harbor, NY: Cold Spring Harbor Laboratory Press.

Harris KM, Stevens JK (1989) Dendritic spines of CA1 pyramidal cells in the rat hippocampus: serial electron microscopy with reference to their biophysical characteristics. J Neurosci 9:2982-2997.

Harris DM, Jensen FE, Tsao BH (1992) Three-dimensional structure of dendritic spines and synapses in rat hippocampus (CA1) at postnatal day 15 and adult ages: implications for the maturation of synaptic physiology and long-term potentiation. J Neurosci 12:2685-2705.

Hosokawa T, Rusakov DA, Bliss TVP, Fine A (1995) Repeated confocal imaging of individual dendritic spines in the living hippocampal slice: evidence for changes in length and orientation associated with chemically induced LTP. J Neurosci 15:5560-5573.
Ishikawa R, Yamashiro S, Matsumura F (1989) Differential modulation of actin-severing activity of gelsolin by multiple isoforms of cultured rat cell tropomyosin. J Biol Chem 264:7490-7497.

Ishikawa R, Hayashi K, Shirao T, Xue Y, Takagi T, Sasaki Y, Kohama K (1994) Drebrin, a development-associated brain protein from rat embryo, causes the dissociation of tropomyosin from actin filaments. J Biol Chem 269:29928-29933.

Kennedy MB, Bennett MK, Erondu NE (1983) Biochemical and immunochemical evidence that the "major postsynaptic density protein" is a subunit of a calmodulin-dependent protein kinase. Proc Natl Acad Sci USA 80:7357-7361.

Kennedy MB, Bennett MK, Bulleit RF, Erondu NE, Jennings VR, Miller SG, Molloy SS, Patton BL, Schenker LJ (1990) Structure and regulation of type II calcium/calmodulin-dependent protein kinase in central nervous system neurons. In: Cold Spring Harbor Symposia on Quantitative Biology, vol LV, pp 101-110. Cold Spring Harbor, NY: Cold Spring Harbor Laboratory.

Koch C, Zador A (1993) The function of dendritic spines: devices subserving biochemical rather than electrical compartmentalization. J Neurosci 13:413-422.

Kodama T, Fukui K, Kometani K (1986) The initial phosphate burst in ATP hydrolysis by myosin and subfragment- 1 as studied by a modified malachite green method for determination of inorganic phosphate. J Biochem 99:1465-1472.

Kojima N, Shirao T, Obata K (1993) Molecular cloning of a developmentally regulated brain protein, chicken drebrin $\mathrm{A}$ and its expression by alternative splicing of the drebrin gene. Mol Brain Res 19:101-114.

Laemmli UK (1970) Cleavage of structural proteins during the assembly of the head of bacteriophage T4. Nature 227:680-685.

Landis DMD, Reese TS (1983) Cytoplasmic organization in cerebellar dendritic spines. J Cell Biol 97:1169-1178.

Lee KS, Schottler F, Oliver M, Lynch G (1980) Brief bursts of highfrequency stimulation produce two types of structural change in rat hippocampus. J Neurophysiol 44:247-258.

Martin LJ, Blackstone CD, Levey AI, Huganir RL, Price DL (1993) AMPA glutamate receptor subunits are differentially distributed in rat brain. Neuroscience 53:327-358.

Morales M, Fifkova E (1989) In situ localization of myosin and actin in dendritic spines with the immunogold technique. J Comp Neurol 279:666-674.

Obata K, Nishiye H, Fujita S, Shirao T, Inoue H, Uchizono K (1986) Identification of a synaptic vesicle-specific 38,000-dalton protein by monoclonal antibodies. Brain Res 375:37-48.

Okagaki T, Higashi-Fujime S, Ishikawa R, Kohama K (1991) In vitro movement of actin filaments on gizzard smooth muscle myosin: requirement of phosphorylation of myosin light chain and effects of tropomyosin and caldesmon. J Biochem (Tokyo) 109:858-866.

Papa M, Bundman MC, Greenberger V, Segal M (1995) Morphological analysis of dendritic spine development in primary cultures of hippocampal neurons. J Neurosci 15:1-11.

Petralia RS, Wang Y-X, Wenthold RJ (1994a) The NMDA receptor subunits NR2A and NR2B show histological and ultrastructural localization patterns similar to those of NR1. J Neurosci 14:6102-6120.

Petralia RS, Yokotani N, Wenthold RJ (1994b) Light and electron microscope distribution of the NMDA receptor subunit NMDAR1 in the rat nervous system using a selective anti-peptide antibody. J Neurosci 14:667-696.

Purpura DP (1974) Dendritic spine "dysgenesis" and mental retardation. Science 186:1126-1128.

Rosenmund C, Westbrook GL (1993) Calcium-induced actin depolymerization reduces NMDA channel activity. Neuron 10:805-814.

Sasaki Y, Hayashi K, Shirao T, Ishikawa R, Kohama K (1996) Inhibition by drebrin of the actin-bundling activity of brain fascin, a protein localized in filopodia of growth cones. J Neurochem 66:980-988.

Shirao T (1995) The roles of microfilament-associated proteins, drebrins, in brain morphogenesis: a review. J Biochem 117:231-236.

Shirao T, Obata K (1985) Two acidic proteins associated with brain development in chick embryo. J Neurochem 44:1210-1216.

Shirao T, Obata K (1986) Immunochemical homology of 3 developmentally regulated brain proteins and their developmental change in neuronal distribution. Dev Brain Res 29:233-244.

Shirao T, Inoue HK, Kano Y, Obata K (1987) Localization of a developmentally regulated neuron-specific protein S54 in dendrites as revealed by immunoelectron microscopy. Brain Res 413:374-378. 
Shirao T, Kojima N, Nabeta Y, Obata K (1989) Two forms of drebrins, developmentally regulated brain proteins, in rat. Proc Jpn Acad Ser B Phys Biol Sci 65:169-172.

Shirao T, Kojima N, Obata K (1992) Cloning of drebrin A and induction of neurite-like processes in drebrin-transfected cells. NeuroReport 3:109-112.

Shirao T, Hayashi K, Ishikawa R, Isa K, Asada H, Ikeda K, Uyemura K (1994) Formation of thick curving bundles of actin by drebrin A expressed in fibroblasts. Exp Cell Res 215:145-153.

Sobue K, Sellers JR (1991) Caldesmon, a novel regulatory protein in smooth muscle and nonmuscle actomyosin systems. J Biol Chem 266:12115-12118.
Tanaka J, Sobue K (1994) Localization and characterization of gelsolin in nervous tissues: gelsolin is specifically enriched in myelin-forming cells. J Neurosci 14:1038-1052.

Tanaka J, Kira M, Sobue K (1993) Gelsolin is localized in neuronal growth cones. Dev Brain Res 76:268-271.

Van Harreveld A, Fifkova E (1975) Swelling of dendritic spines in the fascia dentata after stimulation of the perforant fibers as a mechanism of post-tetanic potentiation. Exp Neurol 49:736-749.

Westrum LE, Jones DH, Gray EG, Barron J (1980) Microtubules, dendritic spines and spine apparatuses. Cell Tissue Res 208:171-181.

Wagner MC, Barylko B, Albanesi JP (1992) Tissue distribution and subcellular localization of mammalian myosin I. J Cell Biol 119:163-170. 